\title{
Pregnancy and birth characteristics of Aboriginal twins in two Australian states: a data linkage study
}

Alison J. Gibberd ${ }^{1 *}$, Jessica Tyler ${ }^{1}$, Kathleen Falster ${ }^{2}$, David B. Preen ${ }^{3}$, Mark Hanly ${ }^{4}$, Marilyn J. Clarke ${ }^{5}$, Bridgette J. McNamara', Sandra J. Eades ${ }^{1,6}$ and Katrina J. Scurrah ${ }^{1}$

\begin{abstract}
Introduction: Perinatal outcomes for singleton pregnancies are poorer, on average, for Aboriginal people than non-Aboriginal people, but little is known about Aboriginal multifetal pregnancies. Yet multifetal pregnancies and births are often more complicated and have poorer outcomes than singleton pregnancies. We describe the pregnancies, births and perinatal outcomes for Aboriginal twins born in Western Australia (WA) and New South Wales (NSW) with comparisons to Aboriginal singletons in both states and to non-Aboriginal births in NSW.

Materials and methods: Whole-population birth records and birth and death registrations were linked for all births during 2000-2013 (WA) and 2002-2008 (NSW). Hospital records and the WA Register of Developmental Anomalies Cerebral Palsy were linked for all WA births and hospital records for a subset of NSW births. Descriptive statistics are reported for maternal and child demographics, maternal health, pregnancy complications, births and perinatal outcomes.

Results: Thirty-four thousand one hundred twenty-seven WA Aboriginal, 32,352 NSW Aboriginal and 601,233 NSW nonAboriginal births were included. Pregnancy complications were more common among mothers of Aboriginal twins than Aboriginal singletons (e.g. 17\% of mothers of WA twins had hypertension/pre-eclampsia/eclampsia vs $8 \%$ of mothers of singletons) but similar to mothers of NSW non-Aboriginal twins. Most Aboriginal twins were born in a principal referral, women's or large public hospital. The hospitals were often far from the mother's home (e.g. 31\% of mothers of WA Aboriginal twins gave birth at hospitals located more than $3 \mathrm{~h}$ by road from their home). Outcomes were worse for Aboriginal liveborn twins than Aboriginal singletons and non-Aboriginal twins (e.g. 58\% of NSW Aboriginal twins were preterm compared to 9\% of Aboriginal singletons and 49\% non-Aboriginal twins).
\end{abstract}

Conclusions: Mothers of Aboriginal twins faced significant challenges during the pregnancy, birth and the postnatal period in hospital and, in addition to accessible specialist medical care, these mothers may need extra practical and psychosocial support throughout their journey.

Keywords: Indigenous, Aboriginal and Torres Strait Islander, Twins, Multiples, Pregnancy, Birth, Perinatal, Linked data

\footnotetext{
* Correspondence: alison.gibberd@unimelb.edu.au

'Melbourne School of Population and Global Health, The University of Melbourne, Parkville, Australia

Full list of author information is available at the end of the article
}

(c) The Author(s). 2021 Open Access This article is licensed under a Creative Commons Attribution 4.0 International License, which permits use, sharing, adaptation, distribution and reproduction in any medium or format, as long as you give appropriate credit to the original author(s) and the source, provide a link to the Creative Commons licence, and indicate if changes were made. The images or other third party material in this article are included in the article's Creative Commons licence, unless indicated otherwise in a credit line to the material. If material is not included in the article's Creative Commons licence and your intended use is not permitted by statutory regulation or exceeds the permitted use, you will need to obtain permission directly from the copyright holder. To view a copy of this licence, visit http://creativecommons.org/licenses/by/4.0/. The Creative Commons Public Domain Dedication waiver (http://creativecommons.org/publicdomain/zero/1.0/) applies to the data made available in this article, unless otherwise stated in a credit line to the data. 


\section{Introduction}

While the majority of Aboriginal and Torres Strait Islander (hereafter respectfully called Aboriginal) mothers and babies have positive pregnancy and birth outcomes, national data continue to show disproportionate adverse outcomes for Aboriginal compared to non-Aboriginal Australians [1]. Aboriginal infants have almost twice the rate of low birth weight, preterm birth and perinatal mortality as non-Aboriginal infants [1]. Poor perinatal health is associated with subsequent development and health [2-4]. To drive improvements, the Coalition of Aboriginal and Torres Strait Islander Peak Organisations and Australian state and federal governments recently set a new Closing the Gap outcome - "Children are born healthy and strong" - with a target of 91\% of Aboriginal liveborn singletons having a healthy birth weight by 2031 [5].

Notably, this new Closing the Gap target excludes multiple births. However, Aboriginal twins and higher multiples may be a particularly high-risk group. Studies in non-Aboriginal populations report that serious complications are more common in multifetal pregnancies than singletons, including anaemia, pre-eclampsia and possibly gestational diabetes, and complications such as twin-to-twin transfusion syndrome are unique to multiple pregnancies with monochorionicity [6, 7]. Multiples are also much more likely than singletons to have adverse birth outcomes such as preterm birth, low birth weight, perinatal mortality and postnatal morbidity [8-10]. Specifically, perinatal mortality rates are approximately 3times higher in twins and 12-times higher in higher-order multiples than singletons [1]. Given these differences, guidelines recommend heightened monitoring of multifetal pregnancies and access to specialist medical care during birth $[11,12]$.

Multiples account for approximately 3\% of babies born each year in Australia [1] yet there is little epidemiological information available on Aboriginal multifetal pregnancies and births. Australia's annual report on pregnancies and births does not separately report on Aboriginal multiples and researchers frequently exclude multiples from analyses [1, 13]. Knowledge of these pregnancies and births is needed to inform the health service response to a population group at high risk of experiencing adverse outcomes. Many Aboriginal mothers live in remote and low socioeconomic status areas, far from the hospitals that provide specialist obstetric and infant care, including neonatal intensive care units (NICUs). For example, all NICUs in the state of Western Australia (WA) are located in Perth, more than $1500 \mathrm{~km}$ from the state's northern-most region, the Kimberley, where $22 \%$ of Aboriginal mothers live (and 1\% of non-Aboriginal mothers) [14]. Furthermore, delayed and less frequent antenatal care is more common among women who experience socioeconomic deprivation or live in remote areas [8].

This paper aims to fill the gap in knowledge of Aboriginal twins by describing the pregnancies, births and perinatal outcomes for all Aboriginal twins born in WA from 2000 to 2013 and New South Wales (NSW) from 2002 to 2008 using linked administrative data, alongside data on Aboriginal singletons in both states and non-Aboriginal births in NSW.

\section{Materials and methods}

\section{Data sources}

Birth records from the Midwives Notification System (WA) and Perinatal Data Collection (NSW), birth registrations, inpatient hospital records from the Hospital Morbidity Data Collection (WA) and Admitted Patients Data Collection (NSW), WA Register of Developmental Anomalies - Cerebral Palsy (WARDA), and death registrations were linked probabilistically by the Data Linkage Branch (WA) and the Centre for Health Record Linkage (NSW). Birth records in both states include deliveries of all live-born or stillborn infants following at least 20 weeks' gestation. Some information could not be reported for both states because it was collected by one state only.

\section{Study population}

WA mothers and babies, 2000-2013, Aboriginal only: The WA study population was all Aboriginal births in WA from 2000 to $2013(N=34,127)$, following exclusion of one infant recorded as a twin who did not link to a corresponding twin. This cohort's data were obtained for the Defying the Odds project [15]. Infants were classified as Aboriginal if they or a parent or grandparent were listed as Aboriginal on their Indigenous Status Flag. This flag assigns Aboriginal status based on a range of administrative records and strikes a balance between under-ascertainment of Aboriginal people and incorrect categorisation of non-Aboriginal people as Aboriginal [16, 17]. Further details regarding the algorithm used to create this flag variable are available in Christensen et al. [18]

NSW mothers and babies, 2000-2008, Aboriginal and non-Aboriginal: The NSW study population included all Aboriginal $(N=32,352)$ and non-Aboriginal $(N=601$, 233) births in NSW from 2002 to 2008, after exclusion of 23 infants recorded as multiples who did not link to corresponding multiples. Additional file 1 provides information about the linkage. Children were classified as Aboriginal if their birth record listed their mother as Aboriginal or their birth registration recorded either parent as Aboriginal. Hospital data were only available for 164,727 (26\%) children (164,588 singletons and twins) who started school and had an Australian Early 
Development Census (AEDC) record in 2009 or 2012 (known as the Seeding Success cohort) [19]. Variables derived using hospital data are reported in Additional file 2 for the subset of children who started school in $2009 / 2012$.

The datasets in this study include many Aboriginal communities in Australia; 45\% of all Aboriginal Australians live in the states of WA and NSW [20].

\section{Analysis variables}

Demographic characteristics: We report maternal age, parity, prior multiple birth (WA only), and private hospital insurance when admitted to hospital for the birth. Quintiles of socio-economic disadvantage were formed using the Australian Bureau of Statistics' Socio-Economic Indexes for Areas Index of Relative Socio-Economic Disadvantage [21]. Further details of disadvantage are in Additional file 1. The remoteness of the mother's residence at the time of the birth was assigned according to the Accessibility/Remoteness Index of Australia Plus (ARIA+) categories (major cities, inner regional, outer regional, remote and very remote) [22].

Maternal health and pregnancy complications: We report whether the mother's first antenatal visit was at or before 14 weeks' completed gestation, after 14 weeks or no antenatal visits. In WA, this information was only available from 2010 onwards. Maternal health conditions included gestational diabetes, pre-existing diabetes, preeclampsia/eclampsia/gestational hypertension, and preexisting hypertension, obtained from the infant's birth record. Additionally, for WA and the NSW 2009/2012 school starters, we also used the mother's birthing admission to obtain diagnoses, as using both birth and hospital records improves classification [23]. Complications of the pregnancy included threatened abortion $(<20$ weeks' gestation), antepartum haemorrhage, threatened preterm labour, and preterm prelabour rupture of membranes. In addition to the birth record, these complications were ascertained from maternal hospital admissions during the pregnancy and birth. The International Statistical Classification of Diseases, Tenth Revision, Australian Modification (ICD-10-AM) [24] codes for these conditions are shown in Additional file 3.

Birth: The hospital where the mother gave birth and transfers from another hospital prior to the birth were identified from hospital records in WA and birth records in NSW. The birth hospital was categorised using peer groups defined by the Australian Institute of Health and Welfare (public principal referral/women's [peer groups D1 and D10], large public [peer groups D2 and D10], small public [remaining public hospitals], private, and born outside hospital) [25]. In both NSW and WA birth records, the small number of births outside hospital are flagged. Estimated travel times by road from the mother's home to the birth hospital are reported. In NSW, births outside hospital with an independent midwife were identified and travel times were set to zero minutes. In WA, home births could not be distinguished from other births outside hospital and travel times were not estimated. Methodological details of the estimation of travel times are outlined in Additional file 1.

Other birth details reported are presentation (vertex, breech and other), labour (spontaneous, induced and no labour), use of instruments and whether the birth was vaginal or caesarean. For WA, we further distinguished between planned and emergency caesareans. WA birth records list whether the birth was attended by an obstetrician, other medical practitioner, midwife, student and/ or no-one/other. NSW birth records list categories of care for the birth as private obstetrician, hospital-based medical, general practitioner, hospital-based midwife, and/or independent midwife.

Perinatal outcomes: These included stillbirth (fetal death after at least 20 completed weeks' gestation), neonatal death (death less than 28 days after a live birth), gestational age, and birth weight. Outcomes of live births included resuscitation and admission to a NICU/special care nursery ( $\mathrm{SCN})$. Length of hospital stay was available for WA and the NSW 2009/2012 school starters and was defined as (a) days from birth to final hospital discharge or (b) for WA 'healthy liveborn' infants with no hospital birth admission record, length of stay listed on the birth record. In WA, infants classified as 'healthy liveborn' infants did not have a birth hospital admission available (57\% of the cohort). 'Healthy liveborn' infants had an ICD-10-AM principal diagnosis code commencing with "Z38".

Cerebral palsy diagnosed by 6 years of age and recorded in the WARDA was also reported. Maternal length of hospital stay was calculated as the number of days from her first admission to her final hospital discharge, including transfers to other (non-psychiatric) hospitals. Postpartum haemorrhage (PPH) requiring blood transfusion has been comprehensively listed in NSW birth records since 2007. In WA, a PPH requiring blood transfusion was indicated if (1) the mother's birthing admission or her child's birth record had a $\mathrm{PPH}$ diagnosis and (b) her birthing admission listed a blood transfusion (ICD-10-AM codes are in Additional file 3).

\section{Statistical analyses}

Counts and proportions are reported for categorical variables. Means and standard deviations or medians and interquartile ranges were calculated for continuous variables. Comparisons were made between: Aboriginal twins and Aboriginal singletons in WA and NSW, nonAboriginal twins and non- Aboriginal singletons in 
NSW, and Aboriginal twins and non-Aboriginal twins in NSW. Pearson's chi-squared test was used to compare proportions for all variables, except remoteness, socioeconomic disadvantage, and maternal and infant length of hospital stay [26]. The Cochran-Armitage test for trend was used for remoteness and socioeconomic disadvantage [27]. Comparisons of length of hospital stay were made using Wilcoxon rank sum tests [26]. The tables include indicators of whether $P$-value $<0.001$ and $P$-value $<0.05$.

\section{Results}

The WA cohort consisted of 33,229 Aboriginal singletons, 880 twins and 18 triplets. In NSW, there were 31, 524 Aboriginal singletons, 794 twins, 30 triplets and 4 quadruplets and 582,355 non-Aboriginal singletons, 18, 338 twins, 516 triplets and 24 quadruplets. Multiples made up 2.6\% of the Aboriginal babies in WA and NSW and $3.1 \%$ of the non-Aboriginal babies in NSW.

\section{Demographic characteristics}

Mothers of twins were at least 1 year older, on average, than mothers of singletons for each of the three groups: Aboriginal WA births, Aboriginal NSW births and nonAboriginal NSW births (Table 1). For Aboriginal births, nulliparity was less common among mothers of twins than mothers of singletons (WA twins: 27\% nulliparous, WA singletons: $32 \%$, NSW twins: $28 \%$, NSW singletons: $35 \%$ ), but for non-Aboriginal NSW infants, nulliparity was similar among mothers of twins and singletons (twins: $44 \%$, singletons: $42 \%$ ). Compared to mothers of singletons, the proportions of mothers of twins living in the most advantaged areas and major cities were 1 to 3 percentage points higher for both Aboriginal and nonAboriginal births.

Comparing mothers of Aboriginal twins and nonAboriginal twins, mothers of Aboriginal twins from both states were at least 4 years younger than mothers of non-Aboriginal twins from NSW and they resided in more remote and disadvantaged areas.

\section{Maternal health and pregnancy complications}

Antenatal care in the first trimester was more common for twin pregnancies than singleton pregnancies for both Aboriginal and non-Aboriginal pregnancies (Table 2). The prevalence of pre-existing conditions was similar for mothers of twins and singletons. Gestational diabetes was also similar at $5 \%$ for mothers of WA Aboriginal singletons and twins, $4 \%$ for NSW Aboriginal singletons and twins, and $5 \%$ for NSW non-Aboriginal singletons and $6 \%$ for non-Aboriginal twins. When both hospital and birth records were used to ascertain gestational diabetes among the NSW 2009/2012 school starters, the proportion of women diagnosed with gestational diabetes was two percentage points higher for twin pregnancies compared to singleton pregnancies (Aboriginal twins: 6\%, Aboriginal singletons: 4\%, non-Aboriginal twins: $8 \%$, non-Aboriginal singletons: 6\%) (Additional file 2, Table S1). Other pregnancy complications were more common among mothers of twins compared to singletons; for example, in WA, pre-eclampsia/eclampsia/gestational hypertension were twice as common in mothers of Aboriginal twins (17\%) compared to mothers of Aboriginal singletons (8\%). These hypertensive disorders were less common in the mothers of NSW babies when only the Perinatal Data Collection diagnoses were considered (Table 1), but similar to the WA cohort when hospital diagnoses were also included for the subset of children who started school in 2009/2012 (Additional file 2, Table S1).

Comparing NSW Aboriginal and non-Aboriginal twin pregnancies, the likelihood of pregnancy complications was similar, with the exception of antepartum haemorrhage among the NSW 2009/2012 school starters (13 and 6\% for Aboriginal and non-Aboriginal twin pregnancies, respectively, and 4 and 3\% for Aboriginal and nonAboriginal singleton pregnancies).

\section{Birth}

The majority of WA Aboriginal twins were born in public principal referral/women's hospitals (65\%) compared to only $26 \%$ of singletons (Table 3 ). In NSW, the most common hospital category for the birth of Aboriginal twins was large hospital (50\%), followed by public principal referral/women's hospital (41\%). For non-Aboriginal twins, the most common hospital category was public principal referral/women's hospital (47\%), followed by private hospital (25\%).

Mothers of twins were more likely to be transferred to another hospital prior to the birth (WA Aboriginal twins and singletons: 13 and 4\%, NSW Aboriginal twins and singletons: 17 and 6\%, and NSW non-Aboriginal twins and singletons: 11 and 2\%). Mothers of Aboriginal twins often gave birth far from home. In WA, almost a third (31\%) of the Aboriginal twin births in hospital were at least $3 \mathrm{~h}$ by road from the mother's home; in NSW, the percentage was $8 \%$.

Caesarean section was the most common method of birth for twins in all three groups and this was more than twice as common for twins than singletons. NSW non-Aboriginal twins were the most likely to be born by caesarean section (63\%), followed by NSW Aboriginal twins (60\%) and WA Aboriginal twins (51\%). 63\% (287/ 453) of WA Aboriginal twin caesarean sections were categorised as emergency caesarean sections.

In WA, twin births were twice as likely to be attended by obstetricians (36\%) as singleton births (17\%). In NSW, Aboriginal twins were much more likely to have a 
Table 1 Demographics of Western Australian (2000-2013) and New South Wales' (2002-2008) births by plurality and Aboriginality

\begin{tabular}{|c|c|c|c|c|c|c|}
\hline \multirow[b]{3}{*}{ Demographics } & \multirow{2}{*}{\multicolumn{2}{|c|}{$\begin{array}{l}\text { Western Australia } \\
(2000-2013) \\
\text { Aboriginal }\end{array}$}} & \multicolumn{4}{|c|}{$\begin{array}{l}\text { New South Wales } \\
(2002-2008)\end{array}$} \\
\hline & & & \multicolumn{2}{|c|}{ Aboriginal } & \multicolumn{2}{|c|}{ Non-Aboriginal } \\
\hline & $\begin{array}{l}\text { Twin } \\
N=880 \\
\mathrm{n}(\%) \\
\end{array}$ & $\begin{array}{l}\text { Singleton } \\
N=33,229 \\
\mathrm{n}(\%) \\
\end{array}$ & $\begin{array}{l}\text { Twin } \\
N=794 \\
\mathrm{n}(\%) \\
\end{array}$ & $\begin{array}{l}\text { Singleton } \\
N=31,524 \\
\mathrm{n}(\%)\end{array}$ & $\begin{array}{l}\text { Twin } \\
N=18,338 \\
\mathrm{n}(\%) \\
\end{array}$ & $\begin{array}{l}\text { Singleton } \\
N=582,355 \\
\mathrm{n}(\%) \\
\end{array}$ \\
\hline \multirow{2}{*}{$\begin{array}{l}\text { Groups compared and statistical } \\
\text { significance reported }\end{array}$} & \multirow[t]{2}{*}{ A } & \multirow[t]{2}{*}{$A$} & B & \multirow[t]{2}{*}{ B } & $C$ & \multirow[t]{2}{*}{$C$} \\
\hline & & & D & & $\mathrm{D}$ & \\
\hline \multicolumn{7}{|l|}{ Maternal characteristics } \\
\hline Age (mean (SD)) & $26.6(5.9)$ & $24.9(6.0)$ & $27.5(6.2)$ & $25.7(6.2)$ & $31.5(5.2)$ & $30.1(5.5)$ \\
\hline \multicolumn{7}{|l|}{ Age group $A B C D$} \\
\hline$<20$ & $112(13)$ & $6909(21)$ & $75(9)$ & $5616(18)$ & $268(1)$ & $17,969(3)$ \\
\hline $20-24$ & $232(26)$ & $10,561(32)$ & $217(27)$ & $9537(30)$ & $1610(9)$ & $79,062(14)$ \\
\hline $25-29$ & $282(32)$ & $8165(25)$ & $208(26)$ & 7662 (24) & $4256(23)$ & $160,857(28)$ \\
\hline $30-34$ & $156(18)$ & $5026(15)$ & $164(21)$ & 5609 (18) & $6893(38)^{2}$ & $198,560(34)$ \\
\hline $35+$ & $98(11)$ & $2560(8)$ & $130(16)$ & $3082(10)$ & $5307(29)^{2}$ & $125,749(22)$ \\
\hline Parity (mean (SD)) & $1.9(1.9)$ & $1.7(1.8)$ & $1.8(1.8)$ & $1.5(1.7)$ & $0.9(1.2)$ & $1.0(1.1)$ \\
\hline \multicolumn{7}{|l|}{ Parity group $A B C D$} \\
\hline 0 & $234(27)$ & $10,773(32)$ & $224(28)$ & $11,058(35)$ & $8120(44)$ & $244,868(42)$ \\
\hline 1 & $220(25)$ & $8352(25)$ & $206(26)$ & $8370(27)$ & $6003(33)^{2}$ & $197,772(34)$ \\
\hline $2+$ & $426(48)$ & $14,104(42)$ & $364(46)$ & $12,024(38)$ & $4181(23)^{2}$ & $138,629(24)$ \\
\hline Previous multiple birth ${ }^{A}$ & $26(3)$ & $465(1)$ & - & - & - & - \\
\hline Insurance at time of birth ${ }^{a}$ & $64(7)$ & $1621(5)$ & - & - & - & - \\
\hline \multicolumn{7}{|l|}{ Socioeconomic disadvantage ${ }^{B C D}$} \\
\hline Quintile 1 (most advantaged) & $64(7)$ & $1946(6)$ & $56(7)$ & $1246(4)$ & $4489(25)$ & $125,066(22)$ \\
\hline Quintile 2 & $82(9)$ & $3083(9)$ & $74(9)$ & $2877(9)$ & $3059(17)$ & $93,835(16)$ \\
\hline Quintile 3 & $156(18)$ & $5249(16)$ & $166(21)$ & 5906 (19) & $3639(20)^{2}$ & $115,981(20)$ \\
\hline Quintile 4 & $182(21)$ & $7612(23)$ & $278(35)$ & $12,240(39)$ & $4115(23)^{2}$ & $138,903(24)$ \\
\hline Quintile 5 (most disadvantaged) & $390(45)$ & $14,778(45)$ & $214(27)$ & $8982(29)$ & $2884(16)$ & $103,054(18)$ \\
\hline \multicolumn{7}{|l|}{ Remoteness of residence ${ }^{a b c D}$} \\
\hline Major cities & $394(45)$ & $14,004(42)$ & $386(49)$ & $14,410(46)$ & $14,702(81)$ & $456,767(79)$ \\
\hline Inner regional & $66(8)$ & $2131(6)$ & $256(32)$ & $10,052(32)$ & $2671(15)^{2}$ & $91,304(16)$ \\
\hline Outer regional & $128(15)$ & $5272(16)$ & $120(15)$ & $5436(17)$ & $761(4) 2$ & $26,874(5)$ \\
\hline Remote & $168(19)$ & $6187(19)$ & $20(3)$ & $1217(4)$ & $52(<1)$ & $1806(<1)$ \\
\hline Very remote & $124(14)$ & $5585(17)$ & $6(1)$ & $136(<1)$ & $0(0)$ & $88(<1)$ \\
\hline \multicolumn{7}{|l|}{ Infant characteristics } \\
\hline \multicolumn{7}{|l|}{ Sex } \\
\hline Male & $450(51)$ & $16,823(51)$ & $404(51)$ & $16,169(51)$ & $9190(50)$ & $299,820(51)$ \\
\hline Female & $430(49)$ & $16,404(49)$ & $390(49)$ & $15,350(49)$ & $9146(50)$ & $282,499(49)$ \\
\hline Same sex twin ${ }^{3}$ & $648(74)$ & - & $546(69)$ & - & $12,252(67)$ & - \\
\hline \multicolumn{7}{|c|}{$\begin{array}{l}\text { SD standard deviation. }{ }^{1} \text { The superscripts }{ }^{A B C D a b c d} \text { which appear next to the demographic factors indicate whether two groups were statistically significantly } \\
\text { different: }{ }^{A} \text { or }{ }^{\mathrm{a}} \text { for a comparison of WA Aboriginal singletons and twins; }{ }^{B} \text { or }{ }^{b} \text { for a comparison of NSW Aboriginal singletons and twins; }{ }^{C} \text { or }{ }^{C} \text { for a comparison } \\
\text { of NSW non-Aboriginal singletons and twins; and }{ }^{D} \text { or }{ }^{d} \text { for a comparison of NSW Aboriginal twins and NSW non-Aboriginal twins. Pearson's chi-squared tests } \\
\text { were conducted for binary variables and Cochran-Armitage trend test for ordinal variables. A capital letter indicates } p<0.001 \text { and a lower-case letter indicates } \\
0.001<p<0.05 .{ }^{2} \text { Some twin pairs had inconsistent information on their two birth records. }{ }^{3} \text { Data were not available on zygosity. Data were missing for: maternal } \\
\text { age }(0 \text { infants in WA, } 180 \text { infants in NSW), maternal parity ( } 0 \text { infants in WA, } 1192 \text { infants in NSW), insurance at time of birth ( } 380 \text { infants in WA), socioeconomic } \\
\text { disadvantage (567 infants in WA, } 5947 \text { infants in NSW), remoteness of residence (50 infants in WA, } 5947 \text { infants in NSW), sex ( } 2 \text { infants in WA, } 43 \text { infants in NSW) } \\
\text { and same sex twin ( } 4 \text { infants - } 2 \text { twin pairs - in WA) }\end{array}$} \\
\hline
\end{tabular}


Table 2 Pregnancies in Western Australia (2000-2013) and New South Wales (2002-2008) by plurality and Aboriginality

\begin{tabular}{|c|c|c|c|c|c|c|}
\hline \multirow[b]{3}{*}{ Characteristic } & \multirow{2}{*}{\multicolumn{2}{|c|}{$\begin{array}{l}\text { Western Australia } \\
(2000-2013) \\
\text { Aboriginal }\end{array}$}} & \multicolumn{4}{|c|}{$\begin{array}{l}\text { New South Wales } \\
(2002-2008)\end{array}$} \\
\hline & & & \multicolumn{2}{|c|}{ Aboriginal } & \multicolumn{2}{|c|}{ Non-Aboriginal } \\
\hline & $\begin{array}{l}\text { Twin } \\
N=880 \\
\mathrm{n}(\%)\end{array}$ & $\begin{array}{l}\text { Singleton } \\
N=33,229 \\
\text { n (\%) }\end{array}$ & $\begin{array}{l}\text { Twin } \\
N=794 \\
\mathrm{n}(\%)\end{array}$ & $\begin{array}{l}\text { Singleton } \\
N=31,524 \\
\mathrm{n}(\%)\end{array}$ & $\begin{array}{l}\text { Twin } \\
N 18,338 \\
\mathrm{n}(\%)\end{array}$ & $\begin{array}{l}\text { Singleton } \\
N=582,355 \\
\mathrm{n}(\%)\end{array}$ \\
\hline \multirow[t]{2}{*}{ Groups compared and statistical significance reported $^{1}$} & $A$ & $A$ & $B$ & $B$ & $C$ & $C$ \\
\hline & & & $\mathrm{D}$ & & $\mathrm{D}$ & \\
\hline \multicolumn{7}{|l|}{ Gestation at first antenatal visit ${ }^{\mathrm{bCD}, 2}$} \\
\hline 13 weeks or less & $120(49)$ & $3797(45)$ & $508(64)$ & $18,955(60)$ & $13,894(76)$ & $410,156(70)$ \\
\hline 14 weeks or more & n.p. & $4551(54)$ & $270(34)$ & $11,545(37)$ & $4244(23)$ & $166,711(29)$ \\
\hline No visit (WA) / No visit or missing (NSW) & n.p. & $125(1)$ & $16(2)$ & $1024(3)$ & $200(1)$ & $5488(1)$ \\
\hline \multicolumn{7}{|l|}{ Maternal health and pregnancy complications } \\
\hline Gestational diabetes $^{\mathrm{Cd}}$ & $48(5)$ & $1724(5)$ & $28(4)$ & $1105 \mathrm{e}(4)$ & $1095(6)^{3}$ & $26,541(5)$ \\
\hline Pre-existing diabetes ${ }^{\mathrm{bcd}}$ & $8(1)$ & $557(2)$ & $12(2)$ & $230(1)$ & $142(1)$ & $3315(1)$ \\
\hline Pre-eclampsia/eclampsia/gestational hypertension ${ }^{\mathrm{ABCd}}$ & $148(17)$ & $2650(8)$ & $69(9)$ & $1618(5)$ & $2087(11)^{3}$ & $31,855(5)$ \\
\hline Pre-existing hypertension ${ }^{C}$ & $18(2)$ & $415(1)$ & $8(1)$ & $286(1)$ & $237(1)^{3}$ & $5547(1)$ \\
\hline Threatened abortion ${ }^{\mathrm{A}}$ & $56(6)$ & $1002(3)$ & - & - & - & - \\
\hline Antepartum haemorrhage ${ }^{A}$ & $84(10)$ & $1787(5)$ & - & - & - & - \\
\hline Threatened preterm labour ${ }^{\mathrm{A}}$ & $256(29)$ & $3099(9)$ & - & - & - & - \\
\hline Preterm prelabour rupture of membranes ${ }^{\mathrm{A}}$ & $174(20)$ & 1695 (5) & - & - & - & - \\
\hline
\end{tabular}

n.p. counts not publishable because of privacy concerns as they are either less than 5 or could lead to calculation of a count of less than $5 .{ }^{1}$ The superscripts ABCDabcd which appear next to the demographic factors indicate whether two groups were statistically significantly different: ${ }^{A}$ or ${ }^{a}$ for a comparison of WA Aboriginal singletons and twins; ${ }^{B}$ or ${ }^{b}$ for a comparison of NSW Aboriginal singletons and twins; ${ }^{C}$ or ${ }^{C}$ for a comparison of NSW non-Aboriginal singletons and twins; and ${ }^{\mathrm{D}}$ or ${ }^{\mathrm{d}}$ for a comparison of NSW Aboriginal twins and NSW non-Aboriginal twins. Pearson's chi-squared tests were conducted. A capital letter indicates $p<0.001$ and a lower-case letter indicates $0.001<p<0.05$. ${ }^{2}$ WA and NSW have different definitions of gestational age at first antenatal visit. In WA, this information is reported by birth hospital and information on antenatal visits outside the hospital may be unavailable. Gestation at first antenatal visit was not recorded in WA until 2010 and data were missing for 25,410 infants in WA. This information was collected in NSW throughout the study period but from 2002 to 2005 mothers who did not receive any antenatal care could not be distinguished from mothers who did receive antenatal care, but their data was missing, so the two groups were combined in NSW. ${ }^{3}$ Some twin pairs had inconsistent information on their two birth records

hospital-based medical model of care (76\%) than Aboriginal singletons (42\%). Private obstetricians were involved in a large proportion of non-Aboriginal NSW twin births (45\%), compared to only $14 \%$ for Aboriginal twin births.

\section{Perinatal outcomes}

Twins were only slightly more likely than singletons to be stillborn, but in both states 3\% of liveborn Aboriginal twins died within 28 days, compared with less than half a percent of Aboriginal singletons (Table 4). Twins were roughly one-kilogram lighter at birth than singletons for both Aboriginal and non-Aboriginal liveborn infants. Aboriginal twins from WA had the lowest mean weight at $2225 \mathrm{~g}$. Among liveborn infants, 35\% of WA Aboriginal twins were born at 37 or more weeks' gestational age compared to $42 \%$ of NSW Aboriginal twins and $51 \%$ of NSW non-Aboriginal twins. Twins were more than twice as likely to be admitted to a NICU/SCN and twin infants and their mothers both averaged at least twice as long in hospital as singleton infants and their mothers. The median length of stay in hospital was 7 days for WA
Aboriginal twins and NSW Aboriginal 2009/2012 school starters twins (interquartile range [IQR]: 4-14 days for WA Aboriginal twins and 4-16 days for NSW Aboriginal twin 2009/2012 school starters) and 6 days [IQR: 48 days] for their mothers (Table 4 and Additional file 2, Table S1). Selected perinatal outcomes for triplets are reported in Additional file 1.

\section{Discussion}

This large population-based study in two Australian states showed that the journey from pregnancy to discharge from hospital presented major challenges for many Aboriginal mothers and their twins. Pregnancy complications, birth interventions, travel far from home and adverse perinatal outcomes were more common for Aboriginal twins than Aboriginal singletons, consistent with data for non-Aboriginal singletons and twins, and more common for Aboriginal twins than non-Aboriginal twins.

As expected, mothers of twins in the Aboriginal and non-Aboriginal cohorts had higher proportions of pregnancy complications than singletons, except for 
Table 3 Birth characteristics in Western Australia (2000-2013) and New South Wales (2002-2008) by plurality and Aboriginality

\begin{tabular}{|c|c|c|c|c|c|c|c|}
\hline \multirow[b]{3}{*}{ Characteristic } & \multirow{2}{*}{\multicolumn{2}{|c|}{$\begin{array}{l}\text { Western Australia } \\
(2000-2013) \\
\text { Aboriginal }\end{array}$}} & \multicolumn{4}{|c|}{$\begin{array}{l}\text { New South Wales } \\
(2002-2008)\end{array}$} & \\
\hline & & & \multicolumn{2}{|l|}{ Aboriginal } & \multicolumn{2}{|c|}{ Non-Aboriginal } & \\
\hline & $\begin{array}{l}\text { Twin } \\
N=880 \\
\mathrm{n}(\%)\end{array}$ & $\begin{array}{l}\text { Singleton } \\
N=33,229 \\
\mathrm{n}(\%)\end{array}$ & $\begin{array}{l}\text { Twin } \\
N=794 \\
\mathrm{n}(\%)\end{array}$ & $\begin{array}{l}\text { Singleton } \\
N=31,524 \\
\mathrm{n}(\%)\end{array}$ & $\begin{array}{l}\text { Twin } \\
N=18,338 \\
\mathrm{n}(\%)\end{array}$ & $\begin{array}{l}\text { Singleton } \\
N=582,355 \\
\mathrm{n}(\%)\end{array}$ & \\
\hline \multirow{2}{*}{$\begin{array}{l}\text { Groups compared and statistical significance } \\
\text { reported }{ }^{1}\end{array}$} & A & A & $B$ & B & C & $C$ & \\
\hline & & & $\mathrm{D}$ & & $\mathrm{D}$ & & \\
\hline \multicolumn{8}{|l|}{ Hospital category for birth ${ }^{A B C D}$} \\
\hline Public principal referral/women's & $573(65)$ & $8608(26)$ & $324(41)$ & $8204(26)$ & $8689(47)$ & $189,260(32)$ & \\
\hline Large public & $141(16)$ & $9397(28)$ & $394(50)$ & $17,171(54)$ & $4595(25)$ & $209,070(36)$ & \\
\hline Medium/small public & $105(12)$ & $12,032(36)$ & $42(5)$ & $4759(15)$ & $318(2)$ & $35,198(6)$ & \\
\hline Private & $54(6)$ & $2730(8)$ & $28(4)$ & $1201(4)$ & $4658(25)$ & $146,078(25)$ & \\
\hline Born outside hospital & $7(1)$ & $345(1)$ & $6(1)$ & $189(1)$ & $78(<1)$ & $2749(<1)$ & \\
\hline Mother transferred prior to birth ${ }^{2, A B C d}$ & $116(13)$ & $1358(4)$ & $40(17)$ & $559(6)$ & $616(11)$ & $3456(2)$ & \\
\hline $\begin{array}{l}\text { Time from home to hospital } \\
\text { (median }(\mathrm{IQR}))^{\mathrm{ABCD}}\end{array}$ & $38(20,401)$ & $19(9,51)$ & $21(11,49)$ & $19(10,39)$ & $20(11,35)$ & $17(11,28)$ & \\
\hline $0-29 \min$ & $324(37)$ & $19,695(60)$ & $440(56)$ & $20,668(66)$ & $12,631(69)$ & $437,239(76)$ & \\
\hline $30-59 \min$ & $146(17)$ & $3110(9)$ & $174(22)$ & $5833(19)$ & $3604(20)$ & $103,700(18)$ & \\
\hline $1 \mathrm{~h}-2 \mathrm{~h}$ & $80(9)$ & $2889(9)$ & $108(14)$ & $3522(11)$ & $1558(9)$ & $31,413(5)$ & \\
\hline $3 \mathrm{~h}$ or greater & $269(31)$ & $4342(13)$ & $66(8)$ & $1227(4)$ & $392(2)$ & $4529(1)$ & \\
\hline $\begin{array}{l}\text { Location of WA private hospital } \\
\text { unknown }\end{array}$ & $54(6)$ & $2730(8)$ & - & - & - & - & \\
\hline \multicolumn{8}{|l|}{ Presentation ${ }^{A B C}$} \\
\hline Vertex & $558(63)$ & $31,693(95)$ & $196(67)$ & $11,957(96)$ & $4532(73)$ & $200,288(96)$ & \\
\hline Breech & 291 (33) & $1165(4)$ & $84(29)$ & $389(3)$ & $1482(24)$ & $7561(4)$ & \\
\hline Other & $31(4)$ & $371(1)$ & $11(4)$ & $97(1)$ & $215(3)$ & $1581(1)$ & \\
\hline \multicolumn{8}{|l|}{ Delivery ${ }^{\mathrm{ABCD}}$} \\
\hline Vaginal birth & $427(49)$ & $25,564(77)$ & $317(40)$ & 24,469 (78) & $6765(37)$ & $421,801(72)$ & \\
\hline Spontaneous labour & $258(29)$ & $19,269(58)$ & $212(27)$ & $18,411(58)$ & $3706(20)$ & $302,880(52)$ & \\
\hline Induced labour & $169(19)$ & $6295(19)$ & $105(13)$ & $6058(19)$ & $3059(17)$ & $118,921(20)$ & \\
\hline Caesarean & $453(51)$ & $7665(23)$ & $477(60)$ & $7022(22)$ & $11,567(63)$ & $160,129(28)$ & \\
\hline \multicolumn{8}{|l|}{ Labour } \\
\hline Spontaneous labour & $134(15)$ & $2279(7)$ & $187(24)$ & $2112(7)$ & $3032(17)$ & $40,546(7)$ & \\
\hline Induced labour & 39 (4) & $1440(4)$ & $16(2)$ & $1225(4)$ & $849(5)$ & $27,946(5)$ & \\
\hline No labour & $280(32)$ & 3946 (12) & 274 (35) & $3685(12)$ & $7686(42)$ & $91,637(16)$ & \\
\hline Planned/emergency & & & - & - & - & - & \\
\hline Planned caesarean & $166(19)$ & $2999(9)$ & - & - & - & - & \\
\hline Emergency caesarean & $287(33)$ & 4666 (14) & - & - & - & - & \\
\hline \multicolumn{8}{|l|}{ Instruments used ${ }^{C d}$} \\
\hline No & $803(91)$ & $30,084(91)$ & $755(95)$ & $29,526(94)$ & $16,912(92)$ & $519,869(89)$ & \\
\hline Yes & $77(9)$ & $3145(9)$ & $39(5)$ & $1973(6)$ & $1420(8)$ & $62,182(11)$ & \\
\hline Accoucheur $(W A)^{3}$ & & & & & & & Model of care (NSW) ${ }^{3}$ \\
\hline Obstetrician ${ }^{\mathrm{A}}$ & $319(36)$ & $5683(17)$ & $42(14)$ & $1298(10)$ & $2818(45)$ & $75,804(36)$ & Private obstetrician ${ }^{\mathrm{bCD}}$ \\
\hline Other medical practitioner ${ }^{\mathrm{A}}$ & $553(63)$ & $8842(27)$ & $223(76)$ & $5274(42)$ & $3274(52)$ & $77,227(37)$ & $\begin{array}{l}\text { Hospital-based } \\
\text { medical }^{\text {bCD }}\end{array}$ \\
\hline
\end{tabular}


Table 3 Birth characteristics in Western Australia (2000-2013) and New South Wales (2002-2008) by plurality and Aboriginality (Continued)

\begin{tabular}{|c|c|c|c|c|c|c|c|}
\hline \multirow[b]{3}{*}{ Characteristic } & \multirow{2}{*}{\multicolumn{2}{|c|}{$\begin{array}{l}\text { Western Australia } \\
(2000-2013) \\
\text { Aboriginal }\end{array}$}} & \multicolumn{4}{|c|}{$\begin{array}{l}\text { New South Wales } \\
(2002-2008)\end{array}$} & \\
\hline & & & \multicolumn{2}{|c|}{ Aboriginal } & \multicolumn{2}{|c|}{ Non-Aboriginal } & \\
\hline & $\begin{array}{l}\text { Twin } \\
N=880 \\
\mathrm{n}(\%)\end{array}$ & $\begin{array}{l}\text { Singleton } \\
N=33,229 \\
\mathrm{n}(\%)\end{array}$ & $\begin{array}{l}\text { Twin } \\
N=794 \\
\mathrm{n}(\%)\end{array}$ & $\begin{array}{l}\text { Singleton } \\
N=31,524 \\
\mathrm{n}(\%)\end{array}$ & $\begin{array}{l}\text { Twin } \\
N=18,338 \\
\mathrm{n}(\%)\end{array}$ & $\begin{array}{l}\text { Singleton } \\
N=582,355 \\
\mathrm{n}(\%)\end{array}$ & \\
\hline Midwife $^{A}$ & $195(22)$ & $19,998(60)$ & $30(10)$ & 2260 (18) & $150(2)$ & 15,807 (8) & General practitioner ${ }^{\mathrm{BCD}}$ \\
\hline Student ${ }^{A}$ & $82(9)$ & 4569 (14) & $192(65)$ & $8948(72)$ & $3570(57)$ & $132,379(63)$ & $\begin{array}{l}\text { Hospital-based } \\
\text { midwife }^{\text {bCd }}\end{array}$ \\
\hline Self/other/unknown & $28(3)$ & $855(3)$ & $0(0)$ & $20(<1)$ & $9(<1)$ & $519(<1)$ & Independent midwife \\
\hline
\end{tabular}

$I Q R$ Interquartile range. ${ }^{1}$ The superscripts ${ }^{A B C D a b c d}$ which appear next to the demographic factors indicate whether two groups were statistically significantly different: ${ }^{A}$ or ${ }^{a}$ for a comparison of WA Aboriginal singletons and twins; ${ }^{B}$ or ${ }^{b}$ for a comparison of NSW Aboriginal singletons and twins; ${ }^{C}$ or ${ }^{C}$ for a comparison of NSW non-Aboriginal singletons and twins; and ${ }^{\mathrm{D}}$ or ${ }^{\mathrm{d}}$ for a comparison of NSW Aboriginal twins and NSW non-Aboriginal twins. Pearson's chi-squared tests were conducted. A capital letter indicates $p<0.001$ and a lower-case letter indicates $0.001<p<0.05$. ${ }^{2}$ Mother transferred prior to birth was only collected comprehensively from 2007 in NSW so only data from 2007 and 2008 births are reported. ${ }^{3}$ Categories of accoucheur (WA) and model of care (NSW) are not mutually exclusive. Data were missing for: hospital category for birth (117 infants in WA, 0 in NSW), mother transferred prior to the birth (434 infants in WA, 440817 in NSW), time from home to hospital (469 infants in WA, 5907 in NSW), presentation (0 infants in WA, 404617 in NSW), delivery (0 infants in WA, 464 in NSW), instruments used ( 0 infants in WA, 335 in NSW), and model of care (404,184 in NSW)

gestational diabetes among non-Aboriginal births. The time period covered by this study largely preceded changes in the diagnostic criteria and testing for gestational diabetes which were progressively implemented in Australian jurisdictions following the publication of the Hyperglycemia and Adverse Pregnancy Outcome (HAPO) study in 2008 [28]. These changes greatly increased diagnoses of gestational diabetes [28] so the proportion of women diagnosed with gestational diabetes in this study (approximately 5\%) is low compared to the national percent of $13.5 \%$ reported recently [1]. Our finding of similar prevalence of gestational diabetes among twin and singleton births in both WA and NSW Aboriginal births and only a slight difference for NSW non-Aboriginal births is consistent with some other studies [29,30], though not all $[7,31,32]$. The slight increase in gestational diabetes for NSW non-Aboriginal mothers of twins may be due to maternal age as gestational diabetes is more common among older women [33] and Egeland and Irgens found that the higher risk for multiple pregnancies was largely attenuated after adjustment for maternal age [32]. It is also possible that some diagnoses are missed for Aboriginal women; a study in remote WA of births in 2013 found that Aboriginal women were less likely to be screened for gestational diabetes with the recommended oral glucose tolerance test [34]. Hypertensive disorders, including pre-eclampsia, eclampsia and gestational hypertension combined, were over twice as common in twin pregnancies, compared to singletons, as found in other populations [35-37]. Prior twin cohort studies have found that hypertensive disorders often onset earlier and have greater severity in twin pregnancies compared to singletons [37, 38].

While pregnancy complications were more common among twin pregnancies compared with singleton pregnancies, percentages were generally similar for NSW
Aboriginal and non-Aboriginal twin pregnancies. However, there was some evidence that gestational diabetes and hypertensive disorders were more common in mothers of non-Aboriginal twins and antepartum haemorrhage was roughly twice as common for mothers of Aboriginal twins (13\%) than non-Aboriginal twins (6\%) in NSW 2009/2012 school starters. This was noteworthy as rates were similar for Aboriginal and non-Aboriginal singleton pregnancies (4 and 3\%, respectively, Table S1) and those singleton rates were in line with findings from other Australian jurisdictions [39]. Additionally, another Australian study on (predominantly non-Aboriginal) twin pregnancies found antepartum haemorrhage affected only $5 \%$ of pregnancies [40]. Possible explanations include that this was a chance result in this subsample of NSW Aboriginal twins or that antepartum haemorrhage risk factors which are more common among Aboriginal mothers (for example, increased parity and tobacco use [21]) pose a particularly high risk for twin pregnancies, and further investigation is warranted.

We found that Aboriginal twins had more adverse perinatal outcomes than Aboriginal singletons and nonAboriginal NSW twins. Perinatal death (stillbirth or neonatal death) was higher for twins (compared to singletons) in both states, particularly WA Aboriginal twins. The persistent gap between Aboriginal and nonAboriginal singleton birth weight and gestational age was also apparent among twins [1]. There was marked a difference between the proportion of Aboriginal and non-Aboriginal twins born at 37 weeks' gestation or more (35\% of WA Aboriginal, $42 \%$ of NSW Aboriginal and $51 \%$ of NSW non-Aboriginal twins), despite similar prevalence of pregnancy complications and a greater proportion of mothers of Aboriginal twins aged 20 to 34 years, an age range with lower risk of preterm birth at 
Table 4 Perinatal outcomes in Western Australia (2000-2013) and New South Wales (2002-2008) by plurality and Aboriginality

\begin{tabular}{|c|c|c|c|c|c|c|}
\hline \multirow[b]{3}{*}{ Perinatal outcomes } & \multirow{2}{*}{\multicolumn{2}{|c|}{$\begin{array}{l}\text { Western Australia } \\
(2000-2013) \\
\text { Aboriginal }\end{array}$}} & \multicolumn{4}{|c|}{$\begin{array}{l}\text { New South Wales } \\
(2002-2008)\end{array}$} \\
\hline & & & \multicolumn{2}{|l|}{ Aboriginal } & \multicolumn{2}{|c|}{ Non-Aboriginal } \\
\hline & $\begin{array}{l}\text { Twin } \\
N=880 \\
\mathrm{n}(\%)\end{array}$ & $\begin{array}{l}\text { Singleton } \\
N=33,229 \\
\mathrm{n}(\%)\end{array}$ & $\begin{array}{l}\text { Twin } \\
N=794 \\
\mathrm{n}(\%)\end{array}$ & $\begin{array}{l}\text { Singleton } \\
N=31,524 \\
\mathrm{n}(\%)\end{array}$ & $\begin{array}{l}\text { Twin } \\
N=18,338 \\
\text { n (\%) }\end{array}$ & $\begin{array}{l}\text { Singleton } \\
N=582,355 \\
\mathrm{n}(\%)\end{array}$ \\
\hline \multirow[t]{2}{*}{ Groups compared and statistical significance reported $^{1}$} & A & A & $B$ & $B$ & $C$ & $C$ \\
\hline & & & $\mathrm{D}$ & & $\mathrm{D}$ & \\
\hline All births & $N=880$ & $N=33,229$ & $N=794$ & $N=31,524$ & $N=18,338$ & $N=582,355$ \\
\hline Stillbirth $^{\mathrm{ac}}$ & $20(2)$ & $398(1)$ & $10(1)$ & $262(1)$ & $332(2)$ & $3295(1)$ \\
\hline Live births only & $N=860$ & $N=32,831$ & $N=784$ & $N=31,262$ & $N=18,006$ & $N=579,060$ \\
\hline Neonatal death ${ }^{\mathrm{ABC}}$ & $29(3)$ & $147(<1)$ & $20(3)$ & $105(<1)$ & $294(2)$ & $1182(<1)$ \\
\hline Birth weight (grams) (mean (SD)) & $2225(682)$ & $3207(636)$ & $2311(661)$ & $3283(622)$ & $2436(605)$ & $3419(545)$ \\
\hline \multicolumn{7}{|l|}{ Birth weight bands $A B C D$} \\
\hline$<1000$ & $59(7)$ & $252(1)$ & $35(4)$ & $174(1)$ & $535(3)$ & $1673(<1)$ \\
\hline 1000-1499 & $67(8)$ & $315(1)$ & $67(9)$ & $224(1)$ & $840(5)$ & $2070(<1)$ \\
\hline 1500-1999 & $153(18)$ & $709(2)$ & $101(13)$ & $549(2)$ & $2213(12)$ & $4458(1)$ \\
\hline $2000-2499$ & $240(28)$ & $2292(7)$ & $239(31)$ & $1895(6)$ & $5101(28)$ & $16,228(3)$ \\
\hline $2500-2999$ & $248(29)$ & $7097(22)$ & $251(32)$ & $5970(19)$ & $6477(36)$ & $81,429(14)$ \\
\hline 3000-3499 & $88(10)$ & $11,544(35)$ & $72(9)$ & $10,742(34)$ & $2498(14)$ & $212,821(37)$ \\
\hline $3500+$ & $5(1)$ & $10,622(32)$ & $18(2)$ & $11,695(37)$ & $320(2)$ & $260,131(45)$ \\
\hline Gestational age (median (IQR)) & $36(33,37)$ & $39(38,40)$ & $36(34,37)$ & $39(38,40)$ & $37(35,38)$ & $39(38,40)$ \\
\hline \multicolumn{7}{|l|}{ Gestational age categories $A B C D$} \\
\hline $20-23$ & $22(3)$ & $62(<1)$ & $9(1)$ & $52(<1)$ & $152(1)$ & $479(<1)$ \\
\hline $24-27$ & $30(3)$ & $183(1)$ & $24(3)$ & $139(<1)$ & $345(2)$ & $1200(<1)$ \\
\hline $28-31$ & $68(8)$ & $414(1)$ & $75(10)$ & $261(1)$ & $1001(6)$ & $2712(<1)$ \\
\hline $32-34$ & $198(23)$ & $922(3)$ & $126(16)$ & $743(2)$ & $2658(15)$ & $7137(1)$ \\
\hline $35-36$ & $240(28)$ & $2329(7)$ & $211(27)$ & $1638(5)$ & $4475(25)$ & $18,783(3)$ \\
\hline $37-38$ & $279(32)$ & $10,030(31)$ & $292(38)$ & $7119(23)$ & $8216(46)$ & $124,134(21)$ \\
\hline $39+$ & $23(3)$ & $18,846(57)$ & $31(4)$ & $21,298(68)$ & $861(5)$ & $424,508(73)$ \\
\hline Resuscitation ${ }^{A B C}$ & $449(52)$ & $11,170(34)$ & $455(58)$ & $13,190(42)$ & $10,463(58)$ & $235,578(41)$ \\
\hline Length of stay (median (IQR) in days) $)^{A}$ & $7(4-14)$ & $3(2-4)$ & - & - & - & - \\
\hline Admitted to NICU or $\mathrm{SCN}^{2, A B C d}$ & $452(64)$ & $4343(29)$ & $500(64)$ & $5984(19)$ & $10,521(57)$ & $82,257(14)$ \\
\hline Cerebral palsy & $5(1)$ & $114(<1)$ & - & - & - & - \\
\hline Respiratory distress syndrome of newborns ${ }^{A}$ & $146(17)$ & $883(3)$ & - & - & - & - \\
\hline \multicolumn{7}{|l|}{ Maternal outcomes } \\
\hline $\mathrm{PPH}$ requiring blood transfusion ${ }^{\mathrm{AC}}$ & $36(4)$ & $607(2)$ & $10(3)$ & $403(3)$ & $175(3)$ & $3389(2)$ \\
\hline Mother's length of stay (median (IQR) in days) ${ }^{\mathrm{A}}$ & $6(4-8)$ & $3(2-5)$ & - & - & - & - \\
\hline
\end{tabular}

$S D$ standard deviation, IQR Interquartile range. ${ }^{1}$ The superscripts ${ }^{A B C D a b c d}$ which appear next to the demographic factors indicate whether two groups were statistically significantly different: ${ }^{\mathrm{A}}$ or ${ }^{\mathrm{a}}$ for a comparison of WA Aboriginal singletons and twins; ${ }^{\mathrm{B}}$ or ${ }^{\mathrm{b}}$ for a comparison of NSW Aboriginal singletons and twins; ${ }^{\mathrm{C}}$ or ${ }^{c}$ for a comparison of NSW non-Aboriginal singletons and twins; and ${ }^{D}$ or ${ }^{d}$ for a comparison of NSW Aboriginal twins and NSW non-Aboriginal twins. Pearson's chi-squared tests were conducted for all variables except length of stay. Wilcoxon rank sum tests were conducted for infant and maternal length of stay. A capital letter indicates $p<0.001$ and a lower-case letter indicates a $0.001<p<0.05 .{ }^{2}$ In WA, admission to a NICU or SCN in the hospital the infant was born in and admission to a NICU in any hospital they were transferred to were included (time in a SCN after being transferred to another hospital was not available). In NSW, admission to a NICU or SCN in the hospital the infant was born in was included (admission to a NICU or SCN after transfer was not available). Data were missing for: birth weight ( 0 infants in WA, 286 in NSW), gestational age (45 infants in WA, 433 in NSW), resuscitation (0 infants in WA, 932 in NSW), admitted to NICU or SCN (18,122 infants in WA, 581 in NSW), PPH requiring blood transfusion (0 infants in WA, 401967 in NSW) and mother's length of stay (90 cases in WA). No data were missing for stillbirth, neonatal death, infant length of stay, cerebral palsy or respiratory distress syndrome of newborns 
the population level $[41,42]$. The increased risk of preterm birth for Aboriginal twins may result from factors associated with spontaneous preterm birth which were not explored in this study, such as infection and smoking $[43,44]$. Another explanation may be that although Aboriginal twins were more likely to be born in facilities with greater medical oversight than Aboriginal singletons (a higher percentage of twin births were at principal referral or specialist women's hospitals and obstetricians were more likely to be present), their health care still differed from non-Aboriginal twins.

This study is the first to describe in detail the pregnancies and births of Aboriginal twins, providing baseline data to inform the response of health services to this important population. Many studies exclude multiples from analyses or provide limited information about Aboriginal twins. Our study covered almost half of the Aboriginal Australian population [20] ensuring sufficient twins for meaningful estimates in each state and allowing assessment of whether patterns were consistent in both states. While these communities share some common recent history, including colonisation and its effects, major differences exist within, and between, these states, including cultural and linguistic differences, remoteness and availability of healthcare. Both have large urban populations (40\% of Aboriginal people in WA and 46\% in NSW), but in WA, $24 \%$ of Aboriginal people live in very remote areas, compared to only $1 \%$ in NSW [20].

By using population-based linked data, selection bias common in cohort studies or surveys using primary data collection - was minimised. Limitations of our study include that the data were collected for administrative, not research, purposes and lacked some relevant information such as reasons for giving birth in a distant hospital and the use of assisted reproductive technologies. However, Aboriginal women are much less likely to access assisted reproductive technologies than non-Aboriginal women - a study of 5 Australian states found that only 3\% (9/ 304) of Aboriginal twin pregnancies were the result of assisted reproductive technology, compared to $24 \%$ (2280/9519) of non-Aboriginal mothers [40]. There were also differences in information recorded in WA and NSW, though most data were comparable. Additionally, different methods of identifying Aboriginal infants were used in the two states and some data could only be reported for a subset of NSW births because linked hospital data were not available for all NSW births. While approximate travel times were an indicator of distance from home and community at the time of birth, the mother's actual method of transport to hospital was not recorded. Finally, mothers from border regions may have travelled interstate, as is common practice in the Far West Local Health District of NSW. As such, some information on birth outcomes may be missing from our data and may disproportionately include high-risk multiple births among Aboriginal women.

Our findings highlight that it is more common for the mothers of Aboriginal twins to face a number of practical challenges that may impact on their antenatal care, pregnancy and birth outcomes than mothers of Aboriginal singletons and non-Aboriginal twins; challenges that have major implications for birth planning and the birth experience for mothers of Aboriginal twins. Specialised medical care and NICUs are concentrated in large urban areas and many mothers needed to travel to access that care. We did not have data about the location of antenatal scans and appointments, but travel may also have been necessary during high-risk twin pregnancies. Mothers may have missed valuable support from their family and community for extended periods of time. This travel may also have led to substantial costs for additional antenatal scans, transport, accommodation and, potentially, childcare for older children and reduced work-related income for herself and any accompanying support people. At present, some government support is available for women to travel. For example, NSW women who must travel over $100 \mathrm{~km}$ can access the Isolated Patients Travel and Accommodation Assistance Scheme, although the full costs of travel and accommodation are not covered and food is not subsidised [45].

While all mothers of twins face challenges, the circumstances of many Aboriginal women mean that these challenges may be more difficult to overcome. Aboriginal women report loneliness and distress with relocation for birth [46]. Additionally, costs associated with antenatal care and birth may impact on Aboriginal women who are disproportionately socio-economically disadvantaged following over 200 years of colonisation. Aboriginal women are also more likely to have older children (only 27 and 28\% of mothers of Aboriginal twins in WA and NSW were nulliparous compared to $44 \%$ of mothers of non-Aboriginal twins) and these children may need to travel with their mothers or be cared for by others. Some women may be distressed that their babies are not born on country [46]. Finally, Aboriginal women often access antenatal and postnatal care through primary health care organisations operated by local Aboriginal communities. Births in a distant hospital may require a transfer of care from midwives at these local Aboriginal services to a large, mainstream hospital which may be perceived as less culturally safe and disrupt continuity of care [47].

Given the range of challenges that mothers of twins may face during pregnancy and birth, this group needs more support than mothers of singletons. While Australian State and Federal Governments currently fund services and develop guidelines for specific groups of mothers (for example, teenage mothers, nulliparous mothers, Aboriginal mothers), as far as we are aware, 
there are no targeted supports for parents of twins, let alone mothers of Aboriginal twins who are more likely to live in remote and socioeconomically deprived areas, be younger, and have other children than mothers of non-Aboriginal twins. Aboriginal mothers of twins are a small group, but they are an important high-risk group, and these mothers should be considered for targeted support. Research with parents and Aboriginal community controlled health organisations (particularly in remote areas) and the major hospitals where many Aboriginal twins are born is needed to identify upfront costs during pregnancy for the birth and the impact of these costs, any medical or psychosocial consequences of relocation for birth, whether additional financial or other practical support is needed and, if so, the optimal timing of the support.

\section{Conclusions}

Many Aboriginal twin pregnancies and births are physically and practically challenging and the majority of multiples are born early and small. This study highlights the importance of policies that support health services to meet the practical, financial and psychosocial needs of mothers and families, in addition to meeting their health needs.

\section{Abbreviations}

AEDC: Australian Early Development Census (AEDC); ICD-10-AM: Internationa Statistical Classification of Diseases, Tenth Revision, Australian Modification; IQR: Interquartile range; NICU: Neonatal intensive care unit; NSW: New South Wales; PPH: Post-partum haemorrhage; SCN: Special care nursery; WA: Western Australia

\section{Supplementary Information}

The online version contains supplementary material available at https://doi. org/10.1186/s12884-021-03945-9.

Additional file 1. Additional methodological details.

Additional file 2: Table S1. Characteristics and outcomes of mothers and their singleton and twin infants born in Western Australia from 2000 to 2013 and the New South Wales 2009/2012 school starters by plurality and Aboriginality.

Additional file 3: Table S2. Codes of the International Classification of Diseases, 10th Revision (ICD-10-AM) and Australian Classification of Health Interventions $(\mathrm{ACHI})$ used to identify maternal health conditions and complications during pregnancy from hospital records and Midwives Notification System (MNS, WA) data and Perinatal Data Collection (PDC, NSW).

\section{Acknowledgements}

We wish to thank the staff at the Western Australian Data Linkage Branch and the Midwives Notification System, Hospital Morbidity Data Collection, WA Register of Developmental Anomalies - Cerebral Palsy (WARDA), and WA birth and death registrations. We also thank the NSW Ministry of Health and the NSW Registry of Births, Deaths and Marriages for providing the data and the NSW Centre for Health Record Linkage for linking the datasets. We also acknowledge Twins Research Australia director John Hopper for his support and Twins Research Australia deputy director Kate Murphy for helpful discussions and planning. Finally, we thank members of the Aboriginal Reference Group in Perth and the CBDRH Aboriginal and Torres Strait Islander Maternal and Child Health Reference Group in New South Wales for their contributions to the research questions and interpretation of results.

\section{Authors' contributions}

AG analysed and interpreted the data and wrote the manuscript. JT estimated the road travel times from the mother's residence to the hospital and was a major contributor in writing the manuscript. KF acquired the NSW data and was a major contributor in writing the manuscript. DP, MH and MC made substantial contribution to the design of the work and editing the manuscript. BM and SE acquired the WA data and contributed to the design of the work and editing the manuscript. KS made substantial contribution to the design of the work and the writing of the manuscript. All authors read and approved the final manuscript.

\section{Funding}

The Defying the Odds Study and Seeding Success Study were funded by the Australian National Health and Medical Research Council (NHMRC) (grant no. 1078214 and 1061713). AG, MH and BM received support from the NHMRC Centre for Research Excellence in Aboriginal Adolescent and Child Health (grant no. 1135273). KS and JT received support from the NHMRC Centre for Research Excellence in Twin Research (grant no. 1079102). KF was supported by an NHMRC Early Career Fellowship (grant no. 1016475) and an NHMRC capacity building grant (grant no. 573122)

\section{Availability of data and materials}

The data that support the findings of this study are available from the WA and NSW government agencies, but restrictions apply to the availability of these data, which were used under license for the current study, and so are not publicly available. Data are however available with relevant ethics permissions and approval of the data custodians of each of the datasets used in this study. For information about data access, approval from the data custodians and data linkage, researchers can contact the WA Data Linkage Branch (https://www.datalinkage-wa.org.au/) regarding the Defying the Odds study data and the NSW Centre for Health Record Linkage (http://www. cherel.org.au/) regarding the Seeding Success study data.

\section{Declarations}

\section{Ethics approval and consent to participate}

This study was approved by the Western Australian Aboriginal Health Ethics Committee (HREC 609), the WA Department of Health Human Ethics Committee (DOH-201530), the University of Melbourne Medicine and Dentistry Human Ethics Sub-Committee (\#1851158), NSW Population and Health Services Research Ethics Committee (no. 2014/04/523), the Aboriginal Health and Medical Research Council (AH\&MRC) of NSW Ethics Committee (no. 1031/14), and the Australian National University Human Research Ethics Committee (no. 2014/384). A waiver of participant consent was granted by the committees. All methods were carried out in accordance with relevant guidelines and regulations.

\section{Consent for publication}

Not applicable.

\section{Competing interests}

The authors declare that they have no competing interests.

\section{Author details}

${ }^{1}$ Melbourne School of Population and Global Health, The University of Melbourne, Parkville, Australia. ${ }^{2}$ School of Population Health, The University of New South Wales, Sydney, Australia. ${ }^{3}$ School of Population and Global Health, The University of Western Australia, Crawley, Australia. ${ }^{4}$ Centre for Big Data Research in Health, The University of New South Wales, Sydney, Australia. ${ }^{5}$ Mid North Coast Local Health District, Port Macquarie, Australia. ${ }^{6}$ Curtin Medical School, Curtin University, Perth, Australia.

Received: 26 February 2021 Accepted: 31 May 2021

Published online: 28 June 2021

\section{References}

1. Australian Institute of Health and Welfare. Australia's mothers and babies 2018: in brief. Perinatal statistics series no. 36. Cat. no. PER 108. Canberra: AlHW; 2020. https://www.aihw.gov.au/getmedia/aa54e74a-bda7-4497-93cee0010cb66231/aihw-per-108.pdf.aspx?inline=true. Accessed 7 Jan 2021 
2. Hanly M, Falster K, Chambers G, Lynch J, Banks E, Homaira N, et al. Gestational age and child development at age five in a population-based cohort of Australian Aboriginal and non-Aboriginal children. Paediatr Perinat Epidemiol. 2018;32(1):114-25. https://doi.org/10.1111/ppe.12426.

3. McNamara BJ, Gubhaju L, Chamberlain C, Stanley F, Eades SJ. Early life influences on cardio-metabolic disease risk in Aboriginal populations-what is the evidence? A systematic review of longitudinal and casecontrol studies. Int J Epidemiol. 2012;41(6):1661-82. https://doi.org/10.1 093/ije/dys190.

4. Mathewson KJ, Chow CH, Dobson KG, Pope El, Schmidt LA, Van Lieshout RJ. Mental health of extremely low birth weight survivors: a systematic review and meta-analysis. Psychol Bull. 2017;143(4):347-83. https://doi.org/10.1037/ bul0000091.

5. Coalition of Aboriginal and Torres Strait Islander Peak Organisations and all Australian Governments. Children are born healthy and strong. Canberra: Australian Government; 2020. https://www.closingthegap.gov.au/children-a re-born-healthy-and-strong. Accessed 29 Jan 2021

6. Rao A, Sairam S, Shehata H. Obstetric complications of twin pregnancies. Best Pract Res Clin Obstet Gynaecol. 2004;18(4):557-76. https://doi.org/10.1 016/j.bpobgyn.2004.04.007.

7. Rauh-Hain JA, Rana S, Tamez H, Wang A, Cohen B, Cohen A, et al. Risk for developing gestational diabetes in women with twin pregnancies. J Matern Fetal Neonatal Med. 2009;22(4):293-9. https://doi.org/10.1080/14767050802 663194.

8. Australian Institute of Health and Welfare. Australia's mothers and babies 2017 - in brief. Perinatal statistics series no. 35. Cat. no. PER 100. Canberra: AlHW; 2019. https://www.aihw.gov.au/getmedia/2a0c22a2-ba27-4ba0-ad47ebbe51854cd6/aihw-per-100-in-brief.pdf.aspx?inline=true. Accessed 7 Oct 2019

9. Peter C, Wenzlaff P, Kruempelmann J, Alzen G, Bueltmann E, Gruessner SE. Perinatal morbidity and early neonatal mortality in twin pregnancies. Open J Obstet Gynecol. 2013;3(01):78-89. https://doi.org/10.4236/ojog.2013.31017.

10. Danon D, Sekar R, Hack KE, Fisk NM. Increased stillbirth in uncomplicated monochorionic twin pregnancies: a systematic review and meta-analysis. Obstet Gynecol. 2013;121(6):1318-26. https://doi.org/10.1097/AOG.0b013e31 8292766b.

11. SA Health. Perinatal practice guidelines: twin pregnancy 2018. https://www. sahealth.sa.gov.au/wps/wcm/connect/public+content/sa+health+internet/ clinical+resources/clinical+topics/perinatal/perinatal+practice+guidelines\#T Accessed 29 Aug 2019.

12. FIGO. Good clinical practice advice: management of twin pregnancy. Int J Gynaecol Obstet. 2019;144(3):330-7

13. Shah PS, Zao J, Al-Wassia H, Shah V, Births KSGoDoPL. Pregnancy and neonatal outcomes of Aboriginal women: a systematic review and metaanalysis. Womens Health Issues. 2011;21(1):28-39. https://doi.org/10.1016/j. whi.2010.08.005.

14. Hutchinson MJ, A; Peirce, A. Western Australia's mothers and babies, 2015: 33rd annual report of the Western Australian midwives' notification system Perth: Department of Health, Western Australia; 2019. https://ww2.health.wa. gov.au/ /media/Files/Corporate/Reports\%20and\%20publications/Perinata 1\%20infant\%20and\%20maternal/WA_Mothers_Babies_2015.pdf. Accessed 7 Oct 2019.

15. McNamara B, Gubhaju L, Jorm L, Preen D, Jones J, Joshy G, et al. Exploring factors impacting early childhood health among Aboriginal and Torres Strait islander families and communities: protocol for a population-based cohort study using data linkage (the 'defying the odds' study). BMJ Open. 2018;8(3): e021236. https://doi.org/10.1136/bmjopen-2017-021236

16. Gibberd AJ, Simpson JM, Eades SJ. Use of family relationships improved consistency of identification of Aboriginal people in linked administrative data. J Clin Epidemiol. 2017;90:144-55. https://doi.org/10.1016/j.jclinepi.2017. 06.021.

17. McNamara BJ, Jones J, Shepherd CC, Gubhaju L, Joshy G, McAullay D, et al. Identifying young Aboriginal and Torres Strait Islander children in linked administrative data: a comparison of methods. Int J Popul Data Sci. 2020; 5(1):1100.

18. Christensen D, Davis G, Draper G, Mitrou F, McKeown S, Lawrence D, et al. Evidence for the use of an algorithm in resolving inconsistent and missing indigenous status in administrative data collections. Aust J Soc Issues. 2014; 49(4):423-43. https://doi.org/10.1002/j.1839-4655.2014.tb00322.x.

19. Falster K, Jorgensen M, Hanly M, Banks E, Brownell M, Eades S, et al. Data Resource Profile: Seeding Success: a cross-sectoral data resource for early childhood health and development research in Australian Aboriginal and non-Aboriginal children. Int J Epidemiol. 2017;46(5):1365-j.

20. Australian Bureau of Statistics. Census of Population and Housing - Counts of Aboriginal and Torres Strait Islander Australians: Counts of Aboriginal and Torres Strait Islander peoples from the 2016 Census of Population and Housing, including intercensal changes over time. Canberra: ABS; 2017. https://www.abs.gov.au/statistics/people/aboriginal-and-torres-strait-islanderpeoples/census-population-and-housing-counts-aboriginal-and-torres-straitislander-australians/latest-release\#summary. Accessed 6 Jan 2021

21. Australian Bureau of Statistics. Census of Population and Housing: SocioEconomic Indexes for Areas (SEIFA), Australia - Data only , 2006. Cat. No. 2033.0.55.001 Canberra: Australia Bureau of Statistics; 2008. Available from: https://www.abs.gov.au/AUSSTATS/abs@.nsf/DetailsPage/2033.0.55.0012 006? OpenDocument. Accessed 13 May 2020.

22. Australian Bureau of Statistics. Australian Standard Geographical Classification (ASGC) Remoteness Area Correspondences, 2006. Cat. no. 1216.0.15.003. Canberra: ABS; 2011. https://www.abs.gov.au/A USSTATS/abs@.nsf/DetailsPage/1216.0.15.0032006?OpenDocument. Accessed 24 Jul 2020

23. Lain SJ, Hadfield RM, Raynes-Greenow CH, Ford JB, Mealing NM, Algert CS, et al. Quality of data in perinatal population health databases: a systematic review. Med Care. 2012;50(4):e7-20. https://doi.org/10.1097/MLR.0b013e31 $821 \mathrm{~d} 2 \mathrm{~b} 1 \mathrm{~d}$.

24. Independent Hospital Pricing Authority. Classifications. Sydney: IHPA; 2019. https://www.ihpa.gov.au/what-we-do/classifications. Accessed 9 Feb 2021

25. Australian Institute of Health and Welfare. Australian hospital peer groups. Health services series no. 66. Cat. no. HSE 170. Canberra: AlHW. 2015. https://www.aihw.gov.au/getmedia/79e7d756-7cfe-49bf-b8c0-0bbb0daa243 0/14825.pdf.aspx?inline=true. Accessed 13 Jul 2020

26. Hollander M, Wolfe DA, Chicken E. Nonparametric statistical methods. 3rd ed. Hoboken: Wiley; 2014.

27. Agresti A. Categorical data analysis. Hoboken: Wiley; 2003.

28. Australian Institute of Health and Welfare. Incidence of gestational diabetes in Australia: changing trends. Canberra: AlHW; 2019. https://www.aihw.gov.a u/reports/diabetes/incidence-of-gestational-diabetes-in-australia/contents/ changing-trends. Accessed 9 Feb 2021

29. Henderson CE, Scarpelli S, LaRosa D, Divon MY. Assessing the risk of gestational diabetes in twin gestation. J Natl Med Assoc. 1995;87(10):757-8.

30. Buhling KJ, Henrich W, Starr E, Lubke M, Bertram S, Siebert G, et al. Risk for gestational diabetes and hypertension for women with twin pregnancy compared to singleton pregnancy. Arch Gynecol Obstet. 2003;269(1):33-6. https://doi.org/10.1007/s00404-003-0483-Z

31. Schwartz DB, Daoud Y, Zazula P, Goyert G, Bronsteen R, Wright D, et al. Gestational diabetes mellitus: metabolic and blood glucose parameters in singleton versus twin pregnancies. Am J Obstet Gynecol. 1999;181(4):912-4. https://doi.org/10.1016/S0002-9378(99)70324-8.

32. Egeland $G M$, Irgens LM. Is a multiple birth pregnancy a risk factor for gestational diabetes? Am J Obstet Gynecol. 2001;185(5):1275-6. https://doi. org/10.1067/mob.2001.118853.

33. Australian Institute of Health and Welfare. Incidence of gestational diabetes in Australia. Canberra: AlHW; 2019. https://www.aihw.gov.au/reports/dia betes/incidence-of-gestational-diabetes-in-australia/contents/gestational-dia betes-incidence. Accessed 4 Jan 2020

34. Kirke AB, Atkinson D, Moore S, Sterry K, Singleton S, Roxburgh C, et al. Diabetes screening in pregnancy failing women in rural Western Australia: an audit of oral glucose tolerance test completion rates. Aust J Rural Health 2019;27(1):64-9. https://doi.org/10.1111/ajr.12465.

35. Laine K, Murzakanova G, Sole KB, Pay AD, Heradstveit S, Räisänen S. Prevalence and risk of pre-eclampsia and gestational hypertension in twin pregnancies: a population-based register study. BMJ Open. 2019;9(7): e029908. https://doi.org/10.1136/bmjopen-2019-029908.

36. Kalafat E, Abiola A, Thilaganathan B, Bhide A, Khalil A. The association between hypertension in pregnancy and preterm birth with fetal growth restriction in Singleton and twin pregnancy: use of twin versus singleton charts. J Clin Med. 2020;9(8):2518. https://doi.org/10.3390/jcm9082518.

37. Krotz S, Fajardo J, Ghandi S, Patel A, Keith LG. Hypertensive disease in twin pregnancies: a review. Twin Res Hum Genet. 2002;5(1):8-14. https://doi. org/10.1375/twin.5.1.8

38. Foo J, Mangos G, Brown M. Characteristics of hypertensive disorders in twin versus singleton pregnancies. Pregnancy Hypertens. 2013;3(1):3-9. https:// doi.org/10.1016/j.preghy.2012.05.005. 
39. Australian Institute of Health and Welfare. Antenatal care use and outcomes for Aboriginal and Torres Strait Islander mothers and their babies 2016 2017. Cat. no. IHW 237. Canberra: AlHW; 2020.

40. Wang AY, Safi N, Ali F, Lui K, Li Z, Umstad MP, et al. Neonatal outcomes among twins following assisted reproductive technology: an Australian population-based retrospective cohort study. BMC Pregnancy Childbirth. 2018;18(1):1-7.

41. Chen X-K, Wen SW, Fleming N, Demissie K, Rhoads GG, Walker M. Teenage pregnancy and adverse birth outcomes: a large population based retrospective cohort study. Int J Epidemiol. 2007;36(2):368-73. https://doi. org/10.1093/ije/dyl284.

42. Carolan M, Frankowska D. Advanced maternal age and adverse perinatal outcome: a review of the evidence. Midwifery. 2011;27(6):793-801. https:// doi.org/10.1016/.j.midw.2010.07.006.

43. Agrawal V, Hirsch E. Intrauterine infection and preterm labor. Semin Fetal Neonatal Med. 2012;17(1):12-9. https://doi.org/10.1016/j.siny.2011.09.001.

44. Baecher-Lind L, Wilcox AJ, Miller W. Infections and reproduction. In: Wilcox AJ, editor. Fertility and pregnancy. New York: Oxford University Press; 2010 p. $57-78$.

45. NSW Government. IPTAAS: isolated patients travel and accommodation assistance scheme. For Patients Sydney: NSW Government; 2020. Available from: http://www.iptaas.health.nsw.gov.au/For-patients. Accessed 18 Dec 2020

46. Barclay L, Kornelsen J, Longman J, Robin S, Kruske S, Kildea S, et al. Reconceptualising risk: perceptions of risk in rural and remote maternity service planning. Midwifery. 2016;38:63-70. https://doi.org/10.1016/j.midw.2 016.04.007.

47. Sivertsen N, Anikeeva O, Deverix J, Grant J. Aboriginal and Torres Strait islander family access to continuity of health care services in the first 1000 days of life: a systematic review of the literature. BMC Health Serv Res. 2020; 20(1):1-9.

\section{Publisher's Note}

Springer Nature remains neutral with regard to jurisdictional claims in published maps and institutional affiliations.

Ready to submit your research? Choose BMC and benefit from:

- fast, convenient online submission

- thorough peer review by experienced researchers in your field

- rapid publication on acceptance

- support for research data, including large and complex data types

- gold Open Access which fosters wider collaboration and increased citations

- maximum visibility for your research: over $100 \mathrm{M}$ website views per year

At $\mathrm{BMC}$, research is always in progress.

Learn more biomedcentral.com/submissions 